\title{
Effects of Paliperidone Palmitate on Healthcare Utilization and Costs for Patients with Schizophrenia: A Claim-based Mirror-image Study in South Korea
}

\author{
Dasom Lee ${ }^{1}$, Boung Chul Lee ${ }^{2,3}$, Soo-Hee Choi ${ }^{4,5}$, Do-Hyung Kang ${ }^{1}$, Duk-In Jon ${ }^{6}$, Myung Hun Jung ${ }^{6}$ \\ ${ }^{1}$ Emotional Information and Communication Technology Association, Seoul, ${ }^{2}$ Health Insurance Review and Assessment Service, Wonju, \\ ${ }^{3}$ Department of Psychiatry, Hallym University Hangang Sacred Heart Hospital, College of Medicine, Hallym University, ${ }^{4}$ Department of \\ Psychiatry, Seoul National University Hospital, ${ }^{5}$ Department of Psychiatry, Institute of Human Behavioral Medicine in SNU-MRC, Seoul National \\ University College of Medicine, Seoul, ${ }^{6}$ Department of Psychiatry, Hallym University Sacred Heart Hospital, College of Medicine, Hallym \\ University, Anyang, Korea
}

\begin{abstract}
Objective: Long-acting injectable (LAl) antipsychotics, such as paliperidone palmitate (PP), are known to improve treatment adherence in patients with schizophrenia, which can lead to reductions in relapse and hospitalization rates. However, relatively few studies have demonstrated the economic impact of LAls, especially in Asian populations. Methods: We conducted a claim-based mirror-image study to explore changes in healthcare utilization and associated costs, among 1,272 South Korean patients with schizophrenia (ICD-10-CM code F20), between the 1-year periods before and after the initiation of PP treatment.

Results: Patients accessed outpatient services more frequently after versus before starting PP treatment, with the number of prescription days increasing by $133.45(p<0.0001)$ and the associated costs increasing by USD $1,497.15(p<$ 0.0001). The number of admission days was reduced by 11.33 after starting PP treatment $(p<0.0001)$ and the associated costs were reduced by USD 1,220.75 $(p<0.0001)$. However, admission cost savings were different according to patients' oral drug compliance. The daily dosages for benztropine, procyclidine, and propranolol decreased, showing that there were fewer side-effects after PP-treatment $(p<0.0001)$.

Conclusion: Although the high acquisition cost of PP has been regarded as an obstacle to its clinical use, our results imply that the high prescription costs for PP may be counterbalanced by the reduced admission costs associated with its use. Economic outcomes for patients treated with LAls should be investigated further to help healthcare decision-makers and providers to determine the value of LAls relative to other treatment medications.
\end{abstract}

KEY WORDS: Mirror-image study; Prescriptions; Hospitalization; Cost comparison.

\section{INTRODUCTION}

Schizophrenia is a chronic and devastating mental illness that has been estimated to affect over 21 million people worldwide [1]. The clinical management of schizophrenia has long been recognized as challenging due to patients' propensity for non-adherence to the daily dosing

Received: October 1, 2019 / Revised: December 5, 2019

Accepted: December 18, 2019

Address for correspondence: Myung Hun Jung

Department of Psychiatry, Hallym University Sacred Heart

Hospital, 22 Gwanpyeong-ro 170beon-gil, Dongan-gu, Anyang

14068, Korea

E-mail: mhjung@hallym.or.kr

ORCID: https://orcid.org/0000-0003-2393-3930 required for efficacy of oral atypical antipsychotic (OAT) medications. Previous studies have reported that poor adherence to and low persistence with oral medications increases the relapse rate, number of hospitalizations, and associated healthcare costs in schizophrenia patients [2-7].

With the emphasis on consistent drug treatment to reduce the rates of treatment failure and relapse among schizophrenia patients, long-acting injectable (LAIs) antipsychotics have emerged as an alternative to OAT medications. LAls ensure a more stable level of medication in the blood compared to OAT medications, and can be administered on a biweekly or monthly basis. Paliperidone palmitate (PP), a type of LAI that has only re-

(c) This is an Open-Access article distributed under the terms of the Creative Commons Attribution Non-Commercial License (http://creativecommons.org/licenses/by-nc/4.0) which permits unrestricted non-commercial use, distribution, and reproduction in any medium, provided the original work is properly cited. 
cently become available, is a depot formulation of paliperidone, the $9-\mathrm{OH}$ metabolite of risperidone. PP has a half-life of $25-49$ days and is administered to patients by healthcare professionals on a monthly basis, which aids in treatment adherence and thereby reduces the relapse and hospitalization rates $[8,9]$.

Significant economic benefits of LAls, due to the reductions in hospitalization rates, have recently been demonstrated in populations in the United States (US) and Europe [10-13]. Patients treated with PP had lower mean hospitalization costs (United States dollar [USD] 18,560) compared to those treated with OAT medications (USD 31,505) [10]. However, relatively few studies have explored the economic impact of PP in Asia $[14,15]$. Because the rate of LAI utilization in East Asia (15.3\%) is higher than that in the US (12\%) but much lower than those in many other countries $21.5 \%$ in Belgium, $24.8 \%$ in Australia, 29\% in the United Kingdom, 30.4\% in France, $45 \%$ in Portugal, and 50\% in Austria) [16], studies quantifying the economic benefits of LAls in Asian populations are needed to aid healthcare decision-makers and providers aiming to determine the appropriate most treatments for patients with schizophrenia.

The present study compared healthcare utilization and associated costs among South Korean schizophrenia patients, all of whom were healthcare insurance beneficiaries, between the 1-year periods before and after the initiation of PP treatment using a mirror-image design. Changes in healthcare utilization and costs were analyzed separately for outpatient visits and admissions. In addition, changes in outpatient and admission costs were analyzed separately according to patients' demographic and clinical characteristics. We also compared the use of oral antipsychotics, benztropine, procyclidine, and propranolol before PP treatment and 1-year after PP treatment.

\section{METHODS}

\section{Subjects}

We retrospectively analyzed the medical data of 2,799 patients with schizophrenia. We obtained claims data from the Health Insurance Review and Assessment Service (HIRA) database, which contains medical information for South Korean patients, including health care utilization and costs, over a period of nearly 8 years (from July 2010 to April 2017). Identifying data were deleted from the
HIRA records in accordance with the Act on the Protection of Personal Information Maintained by Public Agencies.

The inclusion criteria were as follows: a diagnosis of schizophrenia (International Classification of Disease 10th Revision Clinical Modification [ICD-10-CM] code F20), age between 19 and 65 years, and receipt of two or more PP injections during the study period. Patients were excluded if the injections had started during admission because there were insufficient data to conduct sensitivity analyses, or if they had incomplete data. Of the total of 2,799 patients, 1,272 were included in this analysis, following exclusion of 1,299 patients who had injections as an admission, 78 due to violation of the age criterion, and 150 due to missing data (Fig. 1).

\section{Design and Variables}

To conduct this mirror-image study, the day on which PP injections commenced was set as the "mirror point"; we then compared the number of days and costs of healthcare utilization (for outpatient prescriptions and admissions) between the 1-year periods pre- and post-PP commencement. The number of outpatient prescription days was calculated as the total number of days on which outpatient visits took place for prescriptions over 1 year, while for (psychiatric) admissions, the total number of days corresponded to the total number of bed days over 1 year. The medical costs for outpatient prescriptions and admissions were calculated in terms of patients' out-of-

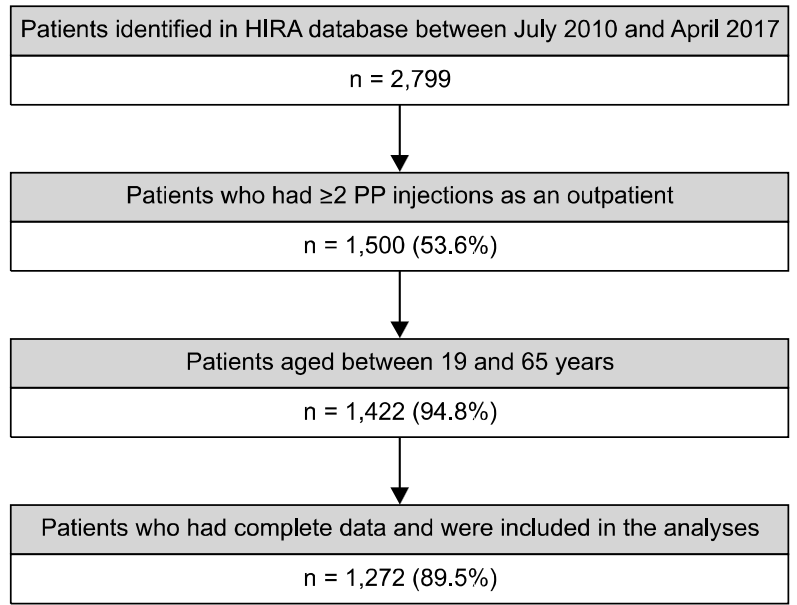

Fig. 1. Enrollment of participants with schizophrenia from the Health Insurance Review and Assessment Service (HIRA) database into the study group.

PP, paliperidone palmitate long-acting injection. 
pocket-costs and national medical insurance costs in Korean won (KRW). All costs were recalculated to USD (KRW 1,000 = USD 0.82 [2019 values]).

To calculate the average daily cost, total costs for outpatient prescriptions and admissions were divided by the number of outpatient prescriptions and admission days, respectively. In addition, a categorical variable that indicated whether patients experienced an admission or made an outpatient visit before and after the start of PP ("Yes" or "No") was included in the analysis.

In order to investigate changes in side effects, we compared the daily dosages of oral antipsychotics, benztropine, procyclidine, and propranolol before PP treatment and 1-year after PP treatment. Because this was a retrospective study, the patients were not required to provide informed consent; the study protocol was approved by the Ethics Committee of Hallym University Sacred Heart Hospital (no. 2019-07-032).

\section{Statistical Analyses}

Statistical analyses were performed using SPSS software (ver. 23.0; IBM Corp., Armonk, NY, USA). First, descriptive statistics on the patients' demographic and clinical characteristics were generated. Second, to evaluate the efficacy of PP, we tested for differences in patterns of healthcare utilization and costs before versus after the mirror point. For categorical measures, chi-squared and McNemar tests were used. For analysis of continuous measures, paired $t$ tests were employed. $p$ values less than 0.05 were considered statistically significant.

\section{RESULTS}

\section{Demographic and Clinical Characteristics}

The mean \pm standard deviation age of the patients was $44.23 \pm 10.30$ years; $44.0 \%$ of the patients were men. The mean \pm standard deviation interval between the first diagnosis of schizophrenia and the start of PP treatment was $5.28 \pm 2.15$ years.

The use of oral antipsychotics during the pre- and post-PP period is described in Table 1. The percentage of patients who were prescribed with oral antipsychotics and their daily dosage were significantly reduced. In addition, the mean number of oral antipsychotics was decreased (Table 1).

Specifically, during the pre-PP period, patients were prescribed with amisulpride $(\mathrm{n}=92)$, aripiprazole $(\mathrm{n}=$ $159)$, chlorpromazine $(n=21)$, clozapine $(n=55)$, haloperidol $(n=43)$, olanzapine $(n=171)$, paliperidone $(n=$ $297)$, perphenazine $(n=5)$, quetiapine $(n=178)$, risperidone $(\mathrm{n}=231)$, sulpiride $(\mathrm{n}=3)$, and ziprasidone $(\mathrm{n}=$ 21).

During the post-PP period, patients were prescribed with amisulpride $(n=47)$, aripiprazole $(n=20)$, chlorpromazine $(n=12)$, clozapine $(n=45)$, haloperidol $(n=11)$, olanzapine $(n=72)$, paliperidone $(n=40)$, perphenazine $(n=2)$, quetiapine $(n=119)$, risperidone $(n=54)$, sulpiride $(\mathrm{n}=0)$, and ziprasidone $(\mathrm{n}=8)$.

\section{Outpatient Prescription Days and Costs}

The percentage of patients who made at least one outpatient visit was $98.9 \%$ (1,258 patients) during the pre-PP period and $100 \%(1,272$ patients $)$ during the post-PP

Table 1. Comparison of oral antipsychotics between pre- and post-PP period $(n=1,149)$

\begin{tabular}{|c|c|c|c|c|}
\hline Use of antipsychotics & Pre-PP period & Post-PP period & $\chi^{2} / \mathrm{t}$ & $p$ value \\
\hline \multicolumn{5}{|l|}{ Use of antipsychotics } \\
\hline Yes & $926(80.6)$ & $348(30.3)$ & $43.31^{b}$ & $<0.0001$ \\
\hline None & $223(19.4)$ & $801(69.7)$ & & \\
\hline Antipsychotics dosage (mg/day) ${ }^{\mathrm{a}}$ & $413.84 \pm 642.02$ & $113.73 \pm 363.48$ & $14.30^{c}$ & $<0.0001$ \\
\hline \multicolumn{5}{|l|}{ Number of antipsychotics } \\
\hline 0 & $223(19.4)$ & $801(69.7)$ & & \\
\hline 1 & $624(54.3)$ & $276(24.0)$ & $142.05^{b}$ & $<0.0001$ \\
\hline$\geq 2$ & $302(26.3)$ & $72(6.3)$ & & \\
\hline
\end{tabular}

Values are presented as number $(\%)$ or mean \pm standard deviation.

$\mathrm{PP}$, paliperidone palmitate long-acting injection; Pre-PP, the 1-year period before the initiation of PP; Post-PP, the 1-year period after the initiation of PP.

${ }^{\mathrm{a}}$ Chlorpromazine equivalent. ${ }^{\mathrm{b}}$ Chi-squared test for categorical variable. ${ }^{\mathrm{c}}$ Paired $t$ test for continuous variable. 
period. The number of days and cost data for outpatient prescriptions are provided in Table 2. Paired $t$ tests showed that patients made more outpatient visits for prescriptions during the post-PP period (332.51 days) than during the pre-PP period (199.06 days, $\mathrm{t}=-33.94, p<$
0.0001; Fig. 2). Outpatient prescription costs increased significantly, from USD 1,505.22 to USD 3,002.37 ( $\mathrm{t}=$ $-34.78, p<0.0001)$. The average daily cost changed from USD 7.56 to USD 9.03.

Table 2. Comparison of healthcare utilization and costs in patients with schizophrenia

\begin{tabular}{|c|c|c|c|c|}
\hline Days and costs & Pre-PP period & Post-PP period & $\mathrm{t}$ & $p$ value \\
\hline \multicolumn{5}{|l|}{ Outpatient $(n=1,272)$} \\
\hline The number of days & $199.06 \pm 134.03$ & $332.51 \pm 59.30$ & -33.94 & $<0.0001$ \\
\hline Total costs (USD) & $1,505.22 \pm 1,353.27$ & $3,002.37 \pm 1,217.12$ & -34.78 & $<0.0001$ \\
\hline \multicolumn{5}{|l|}{ Admission $(n=423)$} \\
\hline The number of days & $54.30 \pm 61.93$ & $29.75 \pm 60.63$ & 5.56 & $<0.0001$ \\
\hline Total costs (USD) & $2,969.53 \pm 3,587.37$ & $1,748.78 \pm 3,639.64$ & 4.82 & $<0.0001$ \\
\hline
\end{tabular}

Values are presented as mean \pm standard deviation.

$\mathrm{PP}$, paliperidone palmitate long-acting injection; Pre-PP, the 1-year period before the initiation of PP; Post-PP, the 1-year period after the initiation of PP; USD, United States dollar.
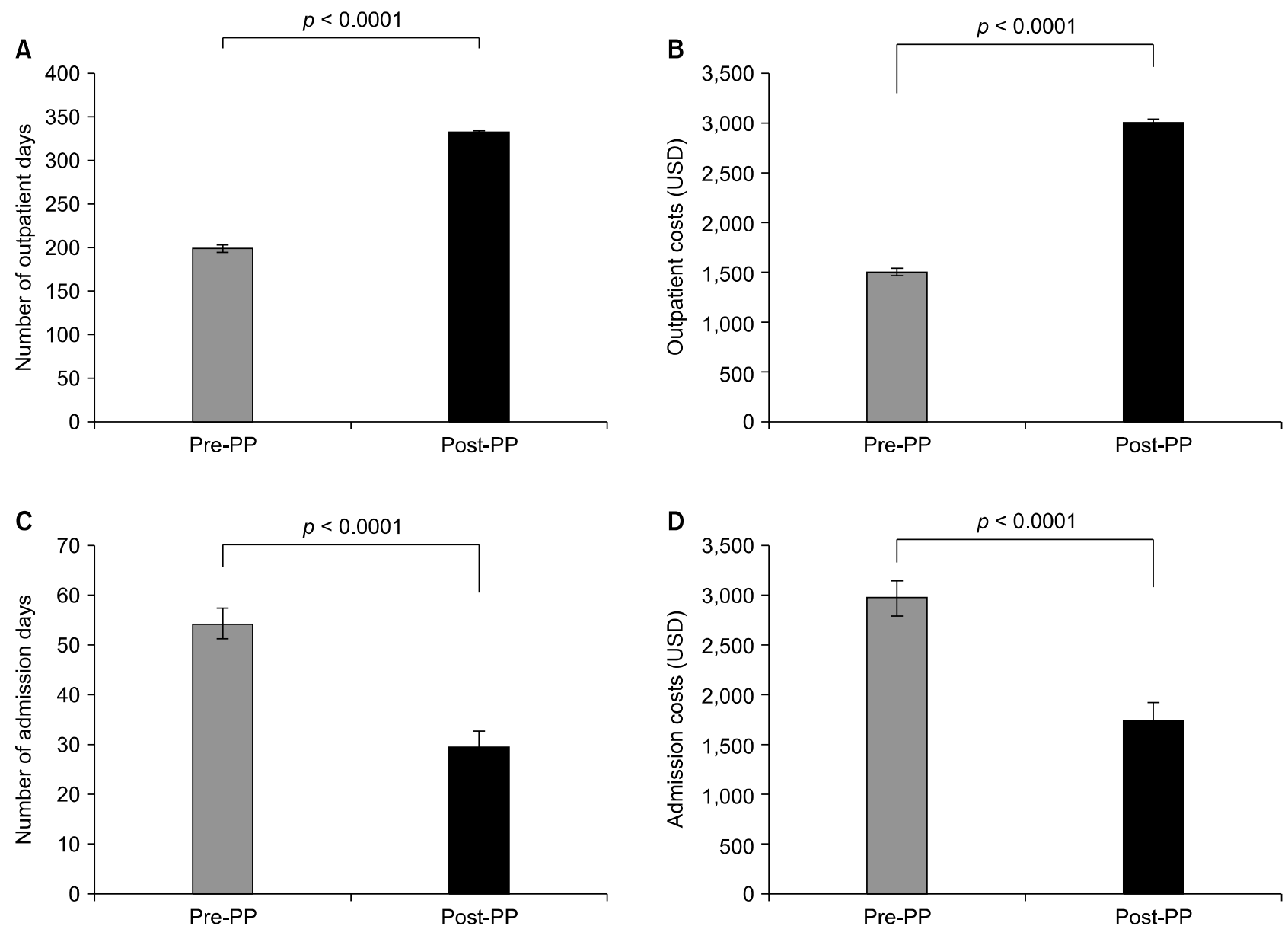

Fig. 2. Number of days and cost data for outpatient prescriptions and admissions for patients with schizophrenia. The data shows an increase in the number of outpatient days (A) with increasing outpatient costs (B), and an decrease in the number of admission days (C) with decreasing admission costs (D).

PP, paliperidone palmitate long-acting injection; Pre-PP, the 1-year period before initiation of PP; Post-PP, the 1-year period after initiation of PP; USD, United States dollar. 


\section{Admission Days and Costs}

The percentage of patients who had experienced an admission decreased from 35.9\% (329 patients) to 19.3\% (177 patients, $p<0.0001$ ) after starting PP treatment. The number of days and cost data for admissions are provided in Table $2(n=423)$. Paired $t$ tests showed that patients had fewer outpatient prescriptions during the post-PP period (29.75 days) than during the pre-PP period (54.30 days, $\mathrm{t}=5.56, p<0.0001$; Fig. 2). Post-PP admission costs (USD 1,748.78) were significantly lower than pre-PP admission costs (USD 2,969.53, $\mathrm{t}=4.82, p<$ $0.0001)$. The average daily cost changed from USD 54.69 to USD 58.78 .

\section{Potential Moderating Variables}

We further investigated whether changes in outpatient and admission costs depend on sex, age, and oral drug compliance (number of outpatient days during the pre-PP period) of patients (Table 3). Outpatient costs after the initiation of PP treatment were significantly increased regardless of sex, age, and oral drug compliance $(p<$ $0.0001)$. In contrast, changes in admission costs were different according to patient's oral drug compliance (but not on sex and age). Admission costs were reduced only for those with low oral drug compliance $(p<0.001)$, but not for those with high oral drug compliance $(p=0.482)$ (Table 3).

\section{Medications for Side Effect}

We conducted additional analyses on the daily dosages of benztropine, procyclidine, and propranolol in order to investigate changes in side effects (Table 4). The daily dosages for all three medications were significantly decreased ( $p<0.0001$ ), implying that patients may have experienced fewer side effects after starting PP treatment compared to before.

Table 4. Comparison of benztropine, procyclidine, propranolol dosage ( $\mathrm{mg} /$ day) between pre- and post-PP period $(\mathrm{n}=1,149)$

\begin{tabular}{cclcc}
\hline \multicolumn{1}{c}{ Drugs } & Pre-PP period & Post-PP period & $\mathrm{t}$ & $p$ value \\
\hline Benztropine & $0.61 \pm 1.37$ & $0.31 \pm 0.79$ & 7.45 & $<0.0001$ \\
Procyclidine & $1.01 \pm 2.74$ & $0.47 \pm 2.19$ & 7.02 & $<0.0001$ \\
Propranolol & $13.26 \pm 46.90$ & $6.78 \pm 21.00$ & 4.97 & $<0.0001$ \\
\hline
\end{tabular}

Values are presented as mean \pm standard deviation.

$\mathrm{PP}$, paliperidone palmitate long-acting injection; Pre-PP, the 1-year period before the initiation of PP; Post-PP, the 1-year period after the initiation of PP.

Table 3. Comparison of healthcare utilization and costs according to patients' demographic and clinical characteristics

\begin{tabular}{|c|c|c|c|c|}
\hline Subgroups & Pre-PP period & Post-PP period & $\mathrm{t}$ & $p$ value \\
\hline \multicolumn{5}{|c|}{ Outpatient costs (USD) $(n=1,272)$} \\
\hline \multicolumn{5}{|c|}{ Number of pre-outpatient days } \\
\hline Low ( $\leq 199$ days) & $1,037.12 \pm 1,207.30$ & $2,788.28 \pm 1,157.05$ & -26.89 & $<0.0001$ \\
\hline High ( > 199 days) & $1,903.81 \pm 1,343.97$ & $3,184.68 \pm 1,237.98$ & -22.88 & $<0.0001$ \\
\hline \multicolumn{5}{|l|}{ Sex } \\
\hline Male & $1,567.57 \pm 1,438.41$ & $3,098.97 \pm 1,271.08$ & -23.60 & $<0.0001$ \\
\hline Female & $1,456.17 \pm 1,281.25$ & $2,926.40 \pm 1,168.24$ & -25.55 & $<0.0001$ \\
\hline \multicolumn{5}{|l|}{ Age (yr) } \\
\hline Age $\leq 45$ & $1,487.18 \pm 1,300.28$ & $2,975.47 \pm 1,323.56$ & -25.36 & $<0.0001$ \\
\hline $45<$ age $\leq 65$ & $1,526.26 \pm 1,413.42$ & $3,033.78 \pm 1,080.02$ & -23.79 & $<0.0001$ \\
\hline \multicolumn{5}{|c|}{ Admission costs (USD) $(n=423)$} \\
\hline \multicolumn{5}{|c|}{ Number of pre-outpatient days } \\
\hline Low ( $\leq 183$ days) & $3,740.52 \pm 3,910.78$ & $1,175.91 \pm 2,977.41$ & 8.04 & $<0.001$ \\
\hline High ( > 183 days) & $2,117.99 \pm 2,977.48$ & $2,381.50 \pm 4,169.98$ & -0.71 & 0.482 \\
\hline \multicolumn{5}{|l|}{ Sex } \\
\hline Male & $3,144.08 \pm 4,000.03$ & $1,871.87 \pm 3,552.86$ & 3.15 & 0.002 \\
\hline Female & $2,840.23 \pm 3,250.69$ & $1,657.60 \pm 3,707.27$ & 3.64 & $<0.001$ \\
\hline \multicolumn{5}{|l|}{ Age (yr) } \\
\hline Age $\leq 45$ & $2,817.43 \pm 3,623.19$ & $1,999.03 \pm 3,966.86$ & 2.37 & 0.019 \\
\hline $45<$ age $\leq 65$ & $3,174.87 \pm 3,538.08$ & $1,410.94 \pm 3,123.49$ & 4.81 & $<0.0001$ \\
\hline
\end{tabular}

Values are presented as mean \pm standard deviation.

$\mathrm{PP}$, paliperidone palmitate long-acting injection; Pre-PP, the 1-year period before the initiation of PP; Post-PP, the 1-year period after the initiation of PP; USD, United States dollar. 


\section{DISCUSSION}

The findings of the present study revealed significant changes in patterns of healthcare utilization and associated costs during the 1-year period after versus before starting PP treatment among South Korean patients with schizophrenia. Patients accessed outpatient services more frequently post-PP commencement; outpatient prescription days increased by 133.45 days and outpatient prescription costs increased by USD 1,497.15. In contrast, the number of admission days decreased by 11.33 days and admission costs were reduced by USD 1,220.75.

Consistent with the findings of the current study, the existing literature includes reports of the efficacy of PP with respect to reducing hospitalization rates and costs in patients with schizophrenia compared to placebo [17] and OAT medications $[4,10,18]$; one of those studies involved a within-patients comparison $[9,19]$. Our results are consistent with prior findings that once-monthly administration of PP enhances treatment adherence, ultimately reducing the economic burden associated with schizophrenia.

Notably, our findings showed that outpatient prescription days and associated costs were increased post-PP commencement. This is because patients consistently visited outpatient clinics to obtain PP, implying that their compliance improved. In a previous 12-week study, patients with schizophrenia showed improved symptoms and reduced side effects, such as extrapyramidal symptoms, when previous OAT medications were replaced with LAls, regardless of the type of OAT medication [20]. Similarly, previous studies have demonstrated that patients treated with LAls made more outpatient visits, for regular administration of injections [19] and because of their transition to outpatient treatment following early discharge from the hospital [14].

The observed increase in outpatient prescription costs may be attributed to the high cost of LAls, which has been regarded as one of the most critical factors limiting clinical use of these drugs. However, previous real-world studies comparing healthcare utilization and costs between LAIs and OAT medications showed that high prescription costs are offset by reduced healthcare expenditure in patients treated with LAls, suggesting that the use of PP may result in greater cost savings compared to the use of OAT medications $[11,12,16]$. Although the present study could not precisely quantify the costs of PP, we observed that a reduction in per patient per year admission costs (USD 1,220.75) could offset the increase in per patient per year prescription costs (USD 1,497.15) in PP-treated Korean patients.

So far, moderating factors in the economic impact of LAls have been rarely investigated. In the present study, we showed that cost savings following the use of PP can depend on patients' oral drug compliance; admission costs were reduced only for those with low oral drug compliance. That is, patients with low oral drug compliance are likely to have greater economic benefits from the PP administration. The admission stays and costs at the time of oral administration might be lower for the patients with high oral drug compliance than patients with low compliance, so there would have been no significant change following the start of PP treatment. Future studies should be conducted to explore more various moderating factors in order to help evaluate the economic benefits of LAls for patients with different clinical characteristics.

We found that patients were prescribed with less benztropine, procyclidine, and propranolol after the start of PP treatment. It is known that long-acting injections can result in more stable serum levels of the drug in the blood, thereby leading to fewer dose-related side effects compared with oral medications [20,21]. In addition, a host of double-blind random clinical trials of PP injection have reported that it can provide an effective treatment for patients with schizophrenia with fewer side effects [22-26]. Consistently, the present study demonstrated that the use of PP injection had positive results in terms of safety.

The results of this study should be interpreted bearing in mind certain limitations. First, we only included patients who started PP injections during outpatient visits, thus excluding those in whom injections were started during admission (because there were insufficient data to conduct sensitivity analyses for the latter patients). Because certain clinical characteristics may differ between these two groups of patients, the generalizability of our findings is likely to have been reduced by exclusion of the latter group. Second, due to the lack of a control group, we cannot unequivocally attribute the observed changes to the effects of PP treatment. Third, outpatient-related variables were not distinguished from each other, such as medical service costs and medication costs.

This retrospective database study demonstrated that, af- 
ter initiation of PP injection treatment, patients accessed outpatient services more frequently. Moreover, the daily cost of outpatient visits was significantly lower than before starting PP, indicating improved treatment adherence. Furthermore, there were fewer hospital stays and lower admission costs. The present results suggest that the high cost of prescribing LAls may be counterbalanced by reduced admission costs. However, healthcare cost savings following the use of PP can depend on patients' clinical characteristics.

\section{Conflicts of Interest}

No potential conflict of interest relevant to this article was reported.

\section{Author Contributions}

Conceptualization: Dasom Lee, Myung Hun Jung. Data acquisition: Boung Chul Lee. Formal analysis: Dasom Lee, Soo-Hee Choi. Supervision: Do-Hyung Kang, Duk-In Jon. Writing - original draft: Dasom Lee. Writing - review \& editing: Boung Chul Lee, Soo-Hee Choi, Do-Hyung Kang, Duk-In Jon, Myung Hun Jung.

\section{ORCID}

Dasom Lee

Boung Chul Lee

https://orcid.org/0000-0003-3776-2263

Soo-Hee Choi https://orcid.org/0000-0002-0968-087X

Do-Hyung Kang https://orcid.org/0000-0003-4507-6108

Duk-In Jon https://orcid.org/0000-0002-7429-2443

Myung Hun Jung https://orcid.org/0000-0002-1565-7940

https://orcid.org/0000-0003-2393-3930

\section{REFERENCES}

1. World Health Organization. World Health Organization health topics: schizophrenia [Internet]. Geneva: World Health Organization; 2015 [cited at 2015 Mar 18]. Available from: http://mww. who.int/mental_health/management/schizophrenia/en/.

2. Chien WT, Yip AL. Current approaches to treatments for schizophrenia spectrum disorders, part I: an overview and medical treatments. Neuropsychiatr Dis Treat 2013;9:13111332.

3. Zhang F, Si T, Chiou CF, Harris AW, Kim CY, Jahagirdar P, et al. Efficacy, safety, and impact on hospitalizations of paliperidone palmitate in recent-onset schizophrenia. Neuropsychiatr Dis Treat 2015;11:657-668.

4. Morrato EH, Parks J, Campagna EJ, Muser E, Thomas DS, Fang $\mathrm{H}$, et al. Comparative effectiveness of injectable paliperidone palmitate versus oral atypical antipsychotics: early postmarketing evidence. J Comp Eff Res 2015;4:89-99.
5. Einarson TR, Geitona M, Chaidemenos A, Karpouza V, Mougiakos T, Paterakis P, et al. Pharmacoeconomic analysis of paliperidone palmitate for treating schizophrenia in Greece. Ann Gen Psychiatry 2012;11:18.

6. Thieda P, Beard S, Richter A, Kane J. An economic review of compliance with medication therapy in the treatment of schizophrenia. Psychiatr Serv 2003;54:508-516.

7. Novick D, Haro JM, Suarez D, Perez V, Dittmann RW, Haddad PM. Predictors and clinical consequences of non-adherence with antipsychotic medication in the outpatient treatment of schizophrenia. Psychiatry Res 2010;176:109-113.

8. Kishimoto T, Nitta M, Borenstein M, Kane JM, Correll CU. Long-acting injectable versus oral antipsychotics in schizophrenia: a systematic review and meta-analysis of mirror-image studies. J Clin Psychiatry 2013;74:957-965.

9. Oh SY, Jon DI, Hong HJ, Hong N, Yi JS, Roh D, et al. The impact of paliperidone palmitate on hospitalization in patients with schizophrenia: a retrospective mirror-image study. Clin Psychopharmacol Neurosci 2019;17:531-536.

10. Baser O, Xie L, Pesa J, Durkin M. Healthcare utilization and costs of Veterans Health Administration patients with schizophrenia treated with paliperidone palmitate long-acting injection or oral atypical antipsychotics. J Med Econ 2015;18: 357-365.

11. Pesa JA, Muser E, Montejano LB, Smith DM, Meyers OI. Costs and resource utilization among Medicaid patients with schizophrenia treated with paliperidone palmitate or oral atypical antipsychotics. Drugs Real World Outcomes 2015;2:377385.

12. Pesa JA, Doshi D, Wang L, Yuce H, Baser O. Health care resource utilization and costs of California Medicaid patients with schizophrenia treated with paliperidone palmitate once monthly or atypical oral antipsychotic treatment. Curr Med Res Opin 2017;33:723-731.

13. Jiang Y, Ni W. Estimating the impact of adherence to and persistence with atypical antipsychotic therapy on health care costs and risk of hospitalization. Pharmacotherapy 2015;35: 813-822.

14. Choon JWY, Wu DBC, Chong HY, Lo WTL, Chong CSY, Chung WS, et al. Real-world evidence of improved healthcare utilization in patients with schizophrenia or schizoaffective disorder after early treatment of paliperidone palmitate once-monthly treatment in Hong Kong. JMed Econ 2019; 22:273-279.

15. Chiou CF, Wang BC, Caldwell R, Furnback W, Lee JS, Kothandaraman $\mathrm{N}$, et al. The cost reduction in hospitalization associated with paliperidone palmitate in the People's Republic of China, Korea, and Malaysia. Neuropsychiatr Dis Treat 2015;11:1989-1994.

16. El Khoury AC, Pilon D, Morrison L, Shak N, Vermette-Laforme $\mathrm{M}$, Amos TB, et al. The prospective economic impact of once monthly paliperidone palmitate versus oral atypical antipsychotics in Medicaid patients with schizophrenia. Curr 
Med Res Opin 2019;35:395-405.

17. Kozma CM, Slaton T, Dirani R, Fastenau J, Gopal S, Hough D. Changes in schizophrenia-related hospitalization and ER use among patients receiving paliperidone palmitate: results from a clinical trial with a 52-week open-label extension (OLE). Curr Med Res Opin 2011;27:1603-1611.

18. Xiao Y, Muser E, Lafeuille MH, Pesa J, Fastenau J, Duh MS, et al. Impact of paliperidone palmitate versus oral atypical antipsychotics on healthcare outcomes in schizophrenia patients. J Comp Eff Res 2015;4:579-592.

19. Ren XS, Crivera C, Sikirica M, Dirani R, Qian S, Kazis LE. Evaluation of health services use following the initiation of risperidone long-acting therapy among schizophrenia patients in the veterans health administration. J Clin Pharm Ther 2011; 36:383-389.

20. Chung S, Cho SJ, Kim CH, Bahk WM, Yoon JS, Chung IW, et al. Effectiveness and tolerability of long-acting risperidone: a 12 weeks, multi-center switching study from oral antipsychotics. Korean J Psychopharmacol 2005;16:109-120.

21. Morris MT, Tarpada SP. Long-acting injectable paliperidone palmitate: a review of efficacy and safety. Psychopharmacol Bull 2017;47:42-52.

22. Bossie CA, Sliwa JK, Ma YW, Fu DJ, Alphs L. Onset of efficacy and tolerability following the initiation dosing of long-acting paliperidone palmitate: post-hoc analyses of a randomized, double-blind clinical trial. BMC Psychiatry 2011;11:79.

23. Gopal S, Hough DW, Xu H, Lull JM, Gassmann-Mayer C, Remmerie BM, et al. Efficacy and safety of paliperidone palmitate in adult patients with acutely symptomatic schizophrenia: a randomized, double-blind, placebo-controlled, dose-response study. Int Clin Psychopharmacol 2010;25: 247-256.

24. Kramer M, Litman R, Hough D, Lane R, Lim P, Liu Y, et al. Paliperidone palmitate, a potential long-acting treatment for patients with schizophrenia. Results of a randomized, double-blind, placebo-controlled efficacy and safety study. Int J Neuropsychopharmacol 2010;13:635-647.

25. Nasrallah HA, Gopal S, Gassmann-Mayer C, Quiroz JA, Lim $\mathrm{P}$, Eerdekens $\mathrm{M}$, et al. A controlled, evidence-based trial of paliperidone palmitate, a long-acting injectable antipsychotic, in schizophrenia. Neuropsychopharmacology 2010;35:20722082.

26. Pandina GJ, Lindenmayer JP, Lull J, Lim P, Gopal S, Herben V, et al. A randomized, placebo-controlled study to assess the efficacy and safety of 3 doses of paliperidone palmitate in adults with acutely exacerbated schizophrenia. J Clin Psychopharmacol 2010;30:235-244. 